\title{
EFEITOS DO USO DE PREBIÓTICOS E PROBIÓTICOS SOBRE OS PARÂMETROS SANGUÍNEOS E NÍVEIS DE ESTRESSE DE FÊMEAS NELORE
}

\author{
André Felipe Berto de Almada ${ }^{1^{*}}$, Monique Catarine Fischer Possamai ${ }^{2}$, Jessé Lahos \\ Borges $^{3}$, Jhordão Rezende Pazianoti ${ }^{4}$, Ranulfo Piau Junior ${ }^{5}$. \\ 1'Doutorando em Ciência Animal com ênfase em produtos bioativos pela \\ Universidade Paranaense, Praça Mascarenhas de Moraes n. 4282, Zona 3. \\ Umuarama - Paraná - Brasil. andrefelipe@zootecnista.com.br. \\ ${ }^{2}$ Mestre em Ciência Animal pela Universidade Paranaense, Praça Mascarenhas de \\ Moraes n. 4282, Zona 3. Umuarama - Paraná - Brasil. \\ ${ }^{3}$ Mestrando em Ciência Animal com ênfase em produtos bioativos pela Universidade \\ Paranaense, Praça Mascarenhas de Moraes n. 4282, Zona 3. Umuarama - Paraná - \\ Brasil. \\ ${ }^{4}$ Academico de Medicina Veterinária pela Universidade Paranaense, Praça \\ Mascarenhas de Moraes n. 4282, Zona 3. Umuarama - Paraná - Brasil. \\ ${ }^{5}$ Docente do programa de pós graduação em Ciência Animal com ênfase em \\ produtos bioativos pela Universidade Paranaense, Praça Mascarenhas de Moraes $\mathrm{n}$. \\ 4282, Zona 3. Umuarama - Paraná - Brasil.
}

\section{Recebido em: 02/10/2017 - Aprovado em: 21/11/2017 - Publicado em: 05/12/2017 DOI: 10.18677/EnciBio_2017B30}

\begin{abstract}
RESUMO
Nos sistemas de produção modernos os animais são submetidos a diferentes graus de estresse devido a condições nutricionais, patológicas, metabólicas ou ambientais. O estresse provoca aumento nos níveis plasmáticos de cortisol, a elevada concentração de cortisol tem efeito imunossupressor deixando os bovinos mais suscetíveis a doenças infecciosas. As 1,3-1,6 betaglucanas são agentes imunomoduladores com potencial na prevenção e controle de doenças infecciosas. O objetivo deste trabalho foi avaliar o efeito do uso de prébiótico e probióticos sobre os parâmetros hematológicos, bioquímicos e níveis de estresse de novilhas confinadas da raça Nelore. Sessenta novilhas da raça Nelore com aproximadamente 24 meses de idade foram distribuídas aleatoriamente formando quatro grupos que receberam prebióticos (PRE), probiótico (PRO), e a simbiose pela associação destes dois aditivos (SIM) e um grupo controle. Os bovinos foram submetidos ao estresse pela prática da manipulação habitual de pesagem no curral, bem como amostragem de sangue nos dias $0,30,60$ e 90. O sangue coletado dos animais com anticoagulante EDTA para análise de hemograma e em frascos com gel para sorologia e bioquímica. Foi desenvolvido um escore de reatividade. Aos 90 dias 0 grupo SIM estava com os níveis elevados de cloretos e mais baixos de fósforo quando comparados com o grupo controle. Não foi encontrada diferença estatística para o ganho de peso. Os aditivos não mostraram diferença estatística na homeostase nos parâmetros bioquímicos e hematológicos. Os animais do grupo probiótico mostraram-se menos reativos a partir do D60.
\end{abstract}

PALAVRAS-CHAVE: Bioativos; Confinamento; Estresse; Reatividade. 


\title{
EFFECTS OF PREBIOTIC AND PROBIOTIC USE ON BLOOD PARAMETERS AND STRESS LEVELS OF NELLORE FEMALES
}

\begin{abstract}
In modern production systems animals undergo different degrees of stress due to nutritional, pathological, metabolic or environmental conditions. Stress causes an increase in plasma cortisol levels, the high concentration of cortisol has an immunosuppressive effect, leaving cattle more susceptible to infectious diseases. 1,3-1,6 beta glucans are immunomodulatory agents with potential in the prevention and control of infectious diseases. The objective of this work was to evaluate the effect of the use of prebiotics and probiotics on the hematological, biochemical and stress levels of heifers of the Nellore breed. Sixty Nelore heifers with approximately 24 months of age were randomly assigned to four groups receiving prebiotics (PRE), probiotic (PRO), and symbiosis by the association of these two additives (SIM) and a control group. The animals were submitted to stress by the practice of the usual manipulation of weighing in the corral, as well as blood sampling on days $0,30,60$ and 90 . Blood collected from the animals with EDTA anticoagulant for blood cell analysis and in flasks with serology gel and biochemistry. A reactivity score was developed. At 90 days the SIM group had high levels of chlorides and lower phosphorus levels when compared to the control group. No statistical difference was found for weight gain. The additives showed no statistical difference in homeostasis in the biochemical and hematological parameters. The animals in the probiotic group were less reactive from $\mathrm{D} 60$.
\end{abstract}

KEYWORDS: Bioactive; Confinement; Reactivity; Stress.

\section{INTRODUÇÃO}

Visando uma forma de vida mais sustentável, a procura por aditivos orgânicos, como por exemplo, os probióticos e prebióticos vêm crescendo, principalmente por profissionais ligados a saúde. As Saccharomyces são leveduras eucarióticas unicelulares, oriundas da fermentação de açucares extraídas nos processos de industrialização da cana-de-açúcar, da indústria cervejeira e do processo de panificação (TOGASHI et al., 2002; CHAUD et al., 2007; MAGNANI; CASTRO-GOMEZ 2008). O cortisol é um ótimo indicativo na relação no nível de estresse, devido a sua atividade antagônica com a produção de anticorpos, no entanto o uso de cromo enriquecido com probióticos produz efeitos positivos no controle do cortisol em vacas zebuínas primíparas (VÁSQUEZ; HERRERA 2003).

Apesar do entendimento de que probióticos e prebióticos causam efeitos benéficos em parâmetros hematológicos e níveis de estresse, os resultados encontrados ainda são controversos, fazendo necessárias mais pesquisas referentes ao assunto (CHIQUIERI et al., 2007; BITTAR et al., 2016). Existem outros produtos que auxiliam no controle da homeostase bovina, como por exemplo, os prebióticos, que favorecem o meio para proliferação dos microrganismos benéficos. Os dois aditivos mais conhecidos deste grupo são os mananoligossacarídeo e as beta-glucanas.

Outro beneficio, desta vez com maior relação no bem estar animal, é no controle do $\mathrm{pH}$ ruminal, podendo chegar em níveis próximos ou até mesmo superiores ao bicarbonato de sódio, mostrando eficiência como tamponante (MARDEN et al., 2008), mostrando ser uma alternativa muito válida para dietas em ENCICLOPÉDIA BIOSFERA, Centro Científico Conhecer - Goiânia, v.14 n.26; p.584 2017 
confinamento. Os níveis sanguíneos de cloro e potássio também servem como parâmetros para níveis de estresse, tendo em vista que o baixo nível de potássio na corrente sanguínea índica que o animal encontra-se fora da sua zona de conforto (JUNQUEIRA et al., 2000).

Segundo Magnani e Castro-Gomez (2008), as beta-glucanas são constituintes da parede celular de fungos presentes em alguns grãos, hoje a principal levedura provedora deste probiótico é a Saccharomyces cerevisiae. A Saccharomyces cerevisiae é um bioativo age como um imunomodulador, promovendo benefícios antitumorais, antimutagênicos, anti-inflamatórios, além do controle do colesterol e glicose (MAGNANI; CASTRO-GOMEZ 2008).

O objetivo deste trabalho foi avaliar o efeito do uso de betaglucanas (prébiótico) e probióticos sobre os parâmetros hematológicos, bioquímicos e níveis de estresse de novilhas confinadas da raça Nelore.

\section{MATERIAL E MÉTODOS}

O experimento foi aprovado pelo Comitê de Ética em Pesquisa Envolvendo Experimentação Animal da Universidade Paranaense, sob 0 protocolo no30166/2016. O experimento foi realizado na fazenda experimental da Universidade Paranaense (Unipar), situada no município de Umuarama, no estado do Paraná, na rodovia PR 482. No período de 25 de julho de 2016 a 24 de outubro de 2016.

Cada baia possui $64 \mathrm{~m}^{2}$. O coxo possui acesso frontal a ração fornecida. Os bebedouros possuem 1,5 metros de comprimento e um metro de largura. Cada baia alojou 15 novilhas para o experimento, deixando uma média de $4,27 \mathrm{~m}^{2}$ por animal e 0,53 metros de linha de cocho.

Sessenta novilhas da raça Nelore com cerca de 24 meses de idade foram distribuídas aleatoriamente formando quatro grupos que receberam prebióticos, probióticos, simbiose destes dois bioativos e um grupo controle. Os animais foram adaptados em um período de uma semana. Todos os animais foram identificados com brincos.

As coletas: peso, reatividade e plasma sanguíneo foram avaliadas de 40 animais, ou seja, 10 animais de cada grupo, escolhidos por sorteio ao acaso. As coletas foram feitas sempre nos mesmos animais a cada 30 dias. Os animais pertenciam ao plantel da fazenda experimental da Universidade Paranaense, aonde se encontravam em regime de recria a pasto, posteriormente foram conduzidas para o sistema de engorda e terminação no manejo de confinamento na fazenda.

Os animais eram arraçoados duas vezes por dia às $08 \mathrm{~h} 00 \mathrm{~min}$ e às $16 \mathrm{~h} 00 \mathrm{~min}$, as sobras foram pesadas semanalmente para estabelecimento da média de consumo por animal dia. As rações continham gérmen de milho, farelo de trigo, resíduo de mandioca e núcleo mineral (Tabela1).

TABELA 1: Composição básica da ração fornecida para as novilhas do confinamento.

\begin{tabular}{lcc}
\hline Ingrediente & Inclusão na matéria seca & Inclusão na matéria natural \\
\hline Gérmen de milho & $15,32 \%$ & $6,0 \%$ \\
Farelo de trigo & $47,84 \%$ & $21,0 \%$ \\
Resíduo de mandioca & $28,61 \%$ & $70,0 \%$ \\
Núcleo & $8,23 \%$ & $3,0 \%$ \\
\hline
\end{tabular}


Os níveis de garantia foram medidos nos parâmetros de matéria seca, proteína bruta, fibra detergente neutro e fibra detergente ácido (Tabela 2).

TABELA 2: Composição bromatológica da ração fornecida para as novilhas do confinamento.

\begin{tabular}{lc}
\hline Descrição & Valores encontrados \\
\hline Matéria Seca & $59,08 \%$ \\
Proteína Bruta & $8,37 \%$ \\
Fibra Detergente Neutro & $23,25 \%$ \\
Fibra Detergente Ácido & $10,91 \%$ \\
\hline
\end{tabular}

Os aditivos utilizados no experimento foram: 1,3 - 1,6 Beta-glucanas como prebiótico, Ruminobacter amylophilum, Ruminobacter succinogenes, Succinovibrio dextrinosol nens, Bacillus cereus, de Lactobacilus acidophilus, Enterococcus faecium e Saccharomyces cerevisiae Saccharomyces cerevisae como probiótico e a associação destes dois aditivos como simbiótico. Foram utilizados aditivos orgânicos, fornecidos logo após o trator arraçoar os cochos, foi utilizado como veiculo para melhor mistura e garantia que todos os animais consumissem a quantidade necessária do aditivo, ou seja 5 gramas de aditivo/animal/dia.

Eram ministrados diariamente três quilos de uma mistura, utilizou-se do gérmen de milho como veiculo na mistura, o consumo diário de cada animal na mistura: veiculo + aditivo era de 200 gramas por dia. No grupo controle os animais consumiam a dieta normal, suplementados com 200 gramas de gérmen de milho. Nos animais tratados com prebióticos (PRE) os animais consumiam 195 gramas de gérmen de milho e 5 gramas de prebióticos, no caso as 1,3-1,6 Beta-glucanas; os animais que foram suplementados com Probióticos (PRO), consumiam 195 gramas de gérmen de milho e 5 gramas de probióticos, então cada animal consumiu: $1,5 \times 10^{9}$ UFC de Ruminobacter amylophilum, $1,5 \times 10^{9}$ de Ruminobacter succinogenes, $2,2 \times 10^{9}$ de Succinovibrio dextrinosol nens, $1,75 \times 10^{9}$ de Bacillus cereus, $1,75 \times 10^{9}$ de Lactobacilus acidophilus, $1,75 \times 10^{9}$ de Enterococcus faecium e $3,75 \times 10^{10}$ de Saccharomyces cerevisiae ; os animais do último grupo foram tratados com prebióticos e probióticos (SIM) nas proporções de 190 gramas de gérmen de milho, 5 gramas de prebióticos e 5 gramas de probióticos.

As coletas eram feitas a cada 30 dias, no inicio (D0), depois de 30 dias foi feita a segunda coleta (D30), 30 dias depois foi feita a terceira coleta (D60) e a última coleta foi feita depois de 30 dias (D90). Os animais eram contidos em tronco, de contenção, com duas travas na região do pescoço, uma no vazio e um protetor de coice. Foi utilizada a porta da parte de traz do tronco para facilitar o acesso à veia coccígea das novilhas.

Para avaliação do nível de estresse acumulado, foram utilizados os seguintes parâmetros: cortisol, glicose, fósforo e cloretos. Foram feitas análises de leucócitos, neutrófilos, linfócitos, hemácias, hemoglobina, hematócrito, proteínas totais, albumina e globulina para avaliar outros parâmetros. Estas análises foram feitas no laboratório de análises clinicas da Universidade Paranaense no aparelho Humanstar 600 para análises dos parâmetros bioquímicos.

As coletas de sangue foram feitas por meio de punção na veia coccígea dos animais do experimento, foram utilizados agulhas hipodérmicas de $40 \times 1,2 \mathrm{~mm}$ e os 
frascos utilizados foram: para hematologia, com anticoagulante EDTA e para sorologia e bioquímica com anticoagulante com gel separador com ativador de coágulo, em seguida as amostras eram agitadas para que não coagulassem e depois armazenadas em caixas térmicas.

As análises de comportamento seguiram o modelo proposto por Aguilar (2007), foi medido: deslocamento (DES) com escores de 1 a 5; postura corporal (PC) com escores de 1 a 3; tensão (TEN) com escores de 1 a 4; respiração (RES) com pontuação de 1 a 3; mugidos (MUG) com pontuação de 0 a 1 e coices (CO) com pontuação de 0 a 1 . Os animais foram mantidos fechados em brete tipo gradil de um metro de largura, 2,12 metros de altura e três metros de comprimento, onde eram mantidos por quatro segundos para análise de reatividade de DES, PC, TEM, RES, MUG E CO, conforme indicado por Aguilar (2007).

Para formação da planilha (Quadro 1) de avaliação foram trabalhados os seguintes escores: DES - nenhum, pouco movimento, deslocamento superior que a metade do tempo, tentar virar e saltar; PC - em pé, ajoelhado e deitado; TEN nenhuma, movimentos bruscos metade do tempo, movimentos bruscos buscando a saída e tremor muscular; RES - normal, audível e bufando; MUG - não e sim e CO - não e sim.

Após a observação do comportamento, as informações eram marcadas em uma ficha impressa, contendo as informações de reatividade pertinente ao trabalho.

Para avaliação da reatividade dos animais foram adaptados alguns parâmetros das ações individuais do gradil: Calmo (DES $=1 ; \mathrm{PC}=1$; $\mathrm{TEN}=1$ ou 2; RES $=1$ ou 2; $\mathrm{MUG}=0$ ou 1 e $\mathrm{CO}=0$ ); Ativo (DES = 1, 2 ou $3 ; \mathrm{PC}=1 ; \mathrm{TEN}=1$ ou $2 ; \mathrm{RES}=1$ ou 2; $M U G=0$ ou 1 e $C O=0$ ou 1); Inquieto (DES = 1, 2 ou 3; $P C=1,2$ ou 3; TEN = 2 ou 3; RES = 1 ou 2; MUG 0 ou 1; CO = 1 ou 1); Perturbado (DES = 3 ou 4; $P C=1$, 2 ou 3; TEN = 2 ou 3; RES = 1, 2 ou 3; $M U G=0$ ou $1 ; \mathrm{CO}=0$ ou 1); Muito Perturbado (DES $=4$ ou 5 ; TEN $=2$ ou 3 independente de outros escores) e Paralisado (TEN $=4$ e DES $=1$ independente de outros escores).

Após os dados serem tabulados em uma planilha de Excel, foi desenvolvido um critério de cálculo para os escores calmo, ativo, inquieto, perturbado, muito perturbado e paralisado, o número de animais $\left(\mathrm{n}^{\circ}\right)$ com cada escore de reatividade foi multiplicado por um valor, em seguida somado e dividido pelo número de animais: Escore de reatividade $=\left(n^{\circ}\right.$ calmos $\left.\times 0\right)+\left(n^{\circ}\right.$ ativos $\left.\times 1\right)+\left(n^{\circ}\right.$ inquietos $\left.\times 2\right)+\left(n^{\circ}\right.$ perturbados $\times 3)+\left(n^{\circ}\right.$ muito perturbados $\left.\times 5\right)+\left(n^{\circ}\right.$ paralisados $\left.\times 5\right) / n^{\circ}$ de animais.

Foram utilizados os números absolutos, não sendo utilizado nenhum método estatístico para estes critérios de reatividade, visando apenas à sugestão de uma metodologia de cálculos. Os dados da coleta de plasma sanguíneo foram analisados no programa Bioestat 5.3 utilizando-se ANOVA fatorial para os dados de ganho de peso e parâmetros sanguíneos, sendo a análise realizada entre os grupos.

\section{RESULTADOS E DISCUSSÃO}

Os níveis de glicose sanguínea apresentaram diferença estatística no D30 entre os grupos SIM e PRO, os níveis do grupo PRO ficaram mais próximos de 75 $\mathrm{ml} / \mathrm{dL}$, que é o valor máximo de referencia (FMVZ 2016). No entanto não houve diferença estatística entre os outros grupos em relação aos dois anteriormente mencionados (Tabela 3). Os níveis de glicose sanguínea foram maiores que parâmetros normais em todos os tratamentos, por todo o período, demonstrando 0 nível de estresse que os animais estavam sendo submetidos durante o manejo e confinamento. 
TABELA 3: Média \pm erro padrão das análises sorológicas de glicose, cortisol, fósforo e cloreto de novilhas confinadas da raça nelore tratadas com probiótico (PRO). prebiótico (PRE) e uma simbiose destes dois bioativos (SIM) e grupo controle nos dias D0, D30, D60, D90.

\begin{tabular}{llcccc}
\hline Grupos & Período & Glicose $\mathbf{~ m g / d L}$ & $\begin{array}{c}\text { Cortisol } \\
\mathbf{\mu g} / \mathbf{d L}\end{array}$ & $\begin{array}{c}\text { Fosforo } \\
\mathbf{m g} / \mathbf{d L}\end{array}$ & $\begin{array}{c}\text { Cloreto } \\
\mathbf{m E q} / \mathbf{L}\end{array}$ \\
\hline Controle & D0 & $112,10 \pm 10,89$ & $3,38 \pm 0,54$ & $7,68 \pm 0,51^{\mathrm{a}}$ & $102,61 \pm 0,51$ \\
PRE & D0 & $113,20 \pm 17,91$ & $2,98 \pm 0,61$ & $6,38 \pm 0,25^{\mathrm{ab}}$ & $103,80 \pm 0,68$ \\
PRO & D0 & $104,90 \pm 9,05$ & $3,23 \pm 0,56$ & $6,00 \pm 0,38^{\mathrm{b}}$ & $101,67 \pm 0,89$ \\
SIM & D0 & $139,00 \pm 12,06$ & $4,09 \pm 0,46$ & $6,32 \pm 0,45^{\mathrm{ab}}$ & $102,13 \pm 0,55$ \\
Controle & D30 & $109,90 \pm 5,98^{\mathrm{ab}}$ & & $8,01 \pm 0,40$ & $96,61 \pm 3,27$ \\
PRE & D30 & $123,20 \pm 14,73^{\mathrm{ab}}$ & & $7,45 \pm 0,40$ & $100,39 \pm 0,52$ \\
PRO & D30 & $106,20 \pm 10,70^{\mathrm{a}}$ & & $8,60 \pm 0,30$ & $97,30 \pm 0,52$ \\
SIM & D30 & $156,30 \pm 15,66^{\mathrm{b}}$ & & $7,90 \pm 0,28$ & $98,30 \pm 0,55$ \\
Controle & D60 & $127,50 \pm 19,60$ & & $8,26 \pm 0,72$ & $96,80 \pm 0,88$ \\
PRE & D60 & $126,00 \pm 19,07$ & & $8,36 \pm 0,27$ & $97,74 \pm 0,67$ \\
PRO & D60 & $118,40 \pm 16,01$ & & $9,77 \pm 0,59$ & $95,20 \pm 0,63$ \\
SIM & D60 & $164,40 \pm 16,29$ & & $7,80 \pm 0,49$ & $97,60 \pm 0,93$ \\
Controle & D90 & $127,0 \pm 18,84$ & $5,82 \pm 0,36$ & $8,13 \pm 0,20^{\mathrm{a}}$ & $97,75 \pm 0,57^{\mathrm{a}}$ \\
PRE & D90 & $97,50 \pm 15,59$ & $4,92 \pm 0,66$ & $7,70 \pm 0,37^{\mathrm{ab}}$ & $98,99 \pm 0,29^{\mathrm{a}}$ \\
PRO & D90 & $104,50 \pm 20,87$ & $5,29 \pm 0,56$ & $7,71 \pm 0,39^{\mathrm{ab}}$ & $99,63 \pm 0,68^{\mathrm{a}}$ \\
SIM & D90 & $116,50 \pm 9,38$ & $5,55 \pm 0,54$ & $6,61 \pm 0,33^{\mathrm{b}}$ & $102,27 \pm 0,47^{\mathrm{b}}$ \\
\hline AnOVa $(P<0,05)$ & & & & & \\
\hline
\end{tabular}

Anova $(\mathrm{P}<0,05)$. Letras diferentes significa diferença estatística entre os grupos.

Os resultados das análises dos níveis de cortisol sanguíneo, que é um parâmetro utilizado para mensuração do estresse nos animais estão na tabela 3. Bittar et al. (2016) em um experimento com bezerros Bos indicus observou maiores níveis de cortisol no plasma sanguíneo do grupo que consumiu um pool de bactérias de ação benéfica no rumem, os autores afirmam que esse resultado se deve ao fato que apenas o grupo tratado recebia o suplemento via oral. É importante salientar que os níveis de cortisol encontrados por Bittar et al. (2016) foram menores que o do presente estudo em todos os tratamentos e em todas as coletas.

Vásquez e Herrera (2003) encontraram resultados favoráveis no controle do cortisol de vacas primíparas suplementadas com cromo usando como veiculo Saccharomyces cerevisae. Apesar do presente estudo não ter encontrado diferenças estatísticas nos níveis de cortisol entre os grupos, podemos observar que os níveis de cortisol em valores absolutos dos grupos PRO e PRE foram mais baixos quando comparados com o grupo controle (Tabela 3 ). 
O fósforo circulante no plasma sanguíneo apresentou níveis superiores aos valores de referência $(5,6-6,5 \mathrm{mg} / \mathrm{dL})$ no início do experimento no grupo controle, e continuaram assim até o final do experimento, os grupos PRO, PRE e SIM começaram o experimento com níveis normais $e$ nas coletas seguintes apresentaram níveis elevados deste mineral, o grupo SIM mostrou grande diminuição no D90, chegando próximo aos valores normais. No D0 foi observada diferença estatística, o grupo controle com índices superiores quando comparados com o grupo PRO e no D90 o grupo controle apresentou diferença estatisticamente superior ao grupo SIM. González et al. (2000) afirmam que os níveis de fósforo presentes na corrente sanguínea está ligado ao desempenho em ganho de peso.

Segundo França e Rodriguez (1997) na fase do estresse em que o indivíduo resiste à adaptação ao meio ocorre uma elevação na produção de cloro, isso se torna importante em animais confinados, estes saem da zona de conforto, do seu habitat natural e podem não se adaptar ao manejo. Os níveis de cloretos do presente estudo mantiveram-se entre $97-111 \mathrm{mg} / \mathrm{dL}$, dentro dos valores de referencia (FMVZ 2016), no entanto encontrou-se diferença estatística nesses parâmetros, no D90 os animais do grupo SIM obtiveram média superior aos animais dos demais grupos, indicando resistência à adaptação ao manejo de confinamento.

Toda alteração sanitária ou mudanças fisiológicas tendem a alterar a atividade das células de defesa nos mamíferos. O hemograma é um bom indicativo de homeostase, pois quando o indivíduo tem seus padrões de saúde afetados tende a ocorrer elevação nos padrões de células de defesa, não foram observadas alterações no hemograma nas novilhas do presente estudo (Tabela 4 e 5).

TABELA 4: Média \pm erro padrão dos valores de leucócitos totais, neutrófilos e linfócitos de novilhas confinadas da raça Nelore tratadas com probiótico (PRO). prebiótico (PRE) e uma simbiose destes dois bioativos (SIM) e grupo controle nos dias D0, D30, D60, D90.

\begin{tabular}{llccc}
\hline Grupos & Período & Leucócitos/ $\mu \mathrm{L}$ & Neutrófilos/ $\boldsymbol{\mu L}$ & Linfócitos/ $\boldsymbol{\mu L}$ \\
\hline Controle & D0 & $7992 \pm 833$ & $2123 \pm 421$ & $5324 \pm 432$ \\
PRE & D0 & $9107 \pm 1254$ & $2686 \pm 205$ & $6564 \pm 703$ \\
PRO & D0 & $1069 \pm 983$ & $2997 \pm 383$ & $7091 \pm 876$ \\
SIM & D0 & $8538 \pm 530$ & $2432 \pm 358$ & $5502 \pm 314$ \\
Controle & D30 & $9466 \pm 680$ & $2472 \pm 0,40$ & $6086 \pm 404$ \\
PRE & D30 & $10503 \pm 599$ & $3079 \pm 318$ & $6497 \pm 587$ \\
PRO & D30 & $17648 \pm 948$ & $2856 \pm 328$ & $7616 \pm 752$ \\
SIM & D30 & $9783 \pm 369$ & $2343 \pm 253$ & $6734 \pm 300$ \\
Controle & D60 & $9624 \pm 586$ & $2568 \pm 317$ & $6379 \pm 374$ \\
PRE & D60 & $11292 \pm 550$ & $3838 \pm 355$ & $6629 \pm 627$ \\
PRO & D60 & $10254 \pm 588$ & $2596 \pm 243$ & $7019 \pm 646$ \\
SIM & D60 & $10589 \pm 456$ & $3082 \pm 341$ & $7100 \pm 627$ \\
Controle & D90 & $9539 \pm 567$ & $2504 \pm 288$ & $6408 \pm 351$ \\
PRE & D90 & $10311 \pm 639$ & $2912 \pm 196$ & $6704 \pm 664$ \\
PRO & D90 & $10642 \pm 740$ & $2778 \pm 201$ & $7153 \pm 726$ \\
SIM & D90 & $10054 \pm 418$ & $2681 \pm 177$ & $6599 \pm 352$ \\
\hline
\end{tabular}

Letras diferentes significa diferença estatística entre os grupos. 
Da mesma forma que o presente trabalho, Chiquieri et al. (2007) também não encontraram diferença estatística nos parâmetros hematológicos de suínos tratados com mananoligossacarídeos como prebióticos, Saccharomyces cerevisea como probióticos, os dois aditivos associados e o grupo controle, no entanto os autores perceberam que o grupo que foi suplementado com mananoligossacarídeos teve médias menores nos leucócitos e linfócitos comparado com os grupos tratados com Saccharomyces cerevisea, os dois aditivos associados e o grupo controle. Resultados próximos aos encontrados com prebióticos no presente estudo.

TABELA 5: Média \pm erro padrão dos valores de Hemácias, hemoglobina e hematócrito de novilhas confinadas da raça Nelore tratadas com probiótico (PRO). prebiótico (PRE) e uma simbiose destes dois bioativos (SIM) e grupo controle nos dias D0, D30, D60, D90.

\begin{tabular}{llccc}
\hline Grupos & Período & Hemácias/milhões & $\begin{array}{c}\text { Hemoglobina } \\
\text { g/DL }\end{array}$ & $\begin{array}{c}\text { Hematócrito } \\
\%\end{array}$ \\
\hline Controle & D0 & $8,28 \pm 0,27$ & $11,59 \pm 0,90$ & $31,86 \pm 1,00$ \\
PRE & D0 & $8,93 \pm 0,36$ & $12,67 \pm 0,46$ & $33,56 \pm 0,84$ \\
PRO & D0 & $8,30 \pm 0,17$ & $11,85 \pm 0,32$ & $31,86 \pm 0,83$ \\
SIM & D0 & $8,72 \pm 0,36$ & $12,02 \pm 0,47$ & $32,56 \pm 1,03$ \\
Controle & D30 & $8,15 \pm 0,23$ & $12,07 \pm 0,30$ & $32,66 \pm 0,75$ \\
PRE & D30 & $8,41 \pm 0,27$ & $12,50 \pm 0,45$ & $33,36 \pm 1,11$ \\
PRO & D30 & $8,28 \pm 0,24$ & $12,346 \pm 0,43$ & $33,21 \pm 1,10$ \\
SIM & D30 & $8,73 \pm 0,36$ & $12,11 \pm 0,44$ & $32,98 \pm 1,09$ \\
Controle & D60 & $8,00 \pm 0,29$ & $11,90 \pm 0,28$ & $32,26 \pm 0,78$ \\
PRE & D60 & $8,38 \pm 0,36$ & $12,53 \pm 0,45$ & $33,42 \pm 1,16$ \\
PRO & D60 & $8,27 \pm 0,19$ & $12,04 \pm 0,30$ & $33,19 \pm 0,88$ \\
SIM & D60 & $8,52 \pm 0,30$ & $12,19 \pm 0,56$ & $32,99 \pm 1,17$ \\
Controle & D90 & $8,21 \pm 0,31$ & $12,48 \pm 0,39$ & $33,08 \pm 1,08$ \\
PRE & D90 & $8,40 \pm 0,36$ & $12,93 \pm 0,59$ & $33,67 \pm 1,51$ \\
PRO & D90 & $8,22 \pm 0,20$ & $12,62 \pm 0,33$ & $32,98 \pm 1,02$ \\
SIM & D90 & $8,52 \pm 0,25$ & $12,25 \pm 0,46$ & $33,20 \pm 1,12$ \\
\hline
\end{tabular}

Letras diferentes significa diferença estatística entre grupos

Corroborando com o presente trabalho, Budiño et al. (2004) em um experimento utilizando Bacilus licheniformes, Bacilus subtiles como probiótico, frutoligossacarídeo como prebiótico e a associação dos dois aditivos como simbiótico na alimentação de suínos, também não encontram diferença estatística entre os grupos nos parâmetros de hemácias, leucócitos, hemoglobina, hematócrito, eosinófilo, neutrófilo bastonete, neutrófilo segmentado, linfócito, monócito e plaqueta. Não houve diferença significativa nos valores de neutrófilos, hemácias, hemoglobina e hematócrito (Tabela 5).

Para os níveis de proteínas totais no plasma sanguíneo, os valores encontrados ficaram entre $7-8,5$, dentro dos padrões de referência para bovinos (FMVZ 2016), no entanto, os índices encontrados para albumina ficaram acima dos padrões durante todo o experimento e os valores de globulina dentro dos padrões para os grupos tratados nos D30 e D90 e o grupo PRE no D60 (Tabela 6). 
TABELA 6: Média \pm erro padrão das análises sorológicas de proteínas totais, albuminas e globulinas de novilhas confinadas da raça Nelore tratadas com probiótico (PRO). prebiótico (PRE) e uma simbiose destes dois bioativos (SIM) e grupo controle nos dias D0, D30, D60, D90.

\begin{tabular}{|c|c|c|c|c|}
\hline Grupos & Período & $\begin{array}{c}\text { Proteínas totais } \\
\text { g/DL }\end{array}$ & $\begin{array}{c}\text { Albuminas } \\
\text { g/DL }\end{array}$ & Globulinas g/DL \\
\hline Controle & D0 & $7,96 \pm 0,31^{a b}$ & $3,48 \pm 0,13$ & $4,47 \pm 0,30^{b}$ \\
\hline PRE & Do & $7,96 \pm 0,18^{a b}$ & $3,79 \pm 0,06$ & $4,16 \pm 0,17^{\mathrm{ab}}$ \\
\hline PRO & Do & $7,34 \pm 0,17^{a}$ & $3,58 \pm 0,15$ & $3,58 \pm 0,15^{a}$ \\
\hline SIM & Do & $8,45 \pm 0,23^{b}$ & $3,90 \pm 0,05$ & $4,55 \pm 0,19^{b}$ \\
\hline Controle & D30 & $7,48 \pm 0,26$ & $3,68 \pm 0,11$ & $3,79 \pm 0,21$ \\
\hline PRE & D30 & $7,17 \pm 0,17$ & $3,70 \pm 0,14$ & $3,48 \pm 0,19$ \\
\hline PRO & $\mathrm{D} 30$ & $7,34 \pm 0,17$ & $3,86 \pm 0,08$ & $3,36 \pm 0,08$ \\
\hline SIM & D30 & $7,14 \pm 0,23$ & $3,93 \pm 0,14$ & $3,31 \pm 0,19$ \\
\hline Controle & $\mathrm{D} 60$ & $7,82 \pm 0,22$ & $4,01 \pm 0,13$ & $3,80 \pm 0,15$ \\
\hline PRE & $\mathrm{D} 60$ & $8,08 \pm 0,17$ & $4,33 \pm 0,11$ & $3,73 \pm 0,11$ \\
\hline PRO & $\mathrm{D} 60$ & $7,83 \pm 0,25$ & $4,34 \pm 0,12$ & $3,48 \pm 0,22$ \\
\hline SIM & D60 & $7,87 \pm 1,12$ & $4,20 \pm 0,11$ & $3,67 \pm 1,12$ \\
\hline Controle & D90 & $7,71 \pm 0,26$ & $4,08 \pm 0,12$ & $3,57 \pm 0,16$ \\
\hline PRE & D90 & $7,61 \pm 0,17$ & $4,29 \pm 0,09$ & $3,31 \pm 0,13$ \\
\hline PRO & D90 & $7,63 \pm 0,20$ & $4,34 \pm 0,09$ & $3,28 \pm 0,18$ \\
\hline SIM & D90 & $7,59 \pm 0,13$ & $4,20 \pm 0,11$ & $3,39 \pm 0,10$ \\
\hline
\end{tabular}

Letras diferentes significa diferença estatística entre grupos

Foi observada diferença estatística nos níveis de globulina apenas no D0, o grupo PRO apresentou valores inferiores comparados com grupo controle. Resultados parecidos foram encontrados por Budiño et al. (2004), Chiquieri et al. (2007), Appelt et al. (2010) e Bittar et al. (2016), que com trabalhos semelhantes com diferentes espécies animais, não encontraram diferença estatística nos níveis de albumina, globulina e/ou proteínas totais no decorrer do experimento.

Todos os tratamentos encontravam-se em estados próximos de reatividade no início do experimento (D0), pode-se perceber que no D30 o grupo controle teve um menor aumento, enquanto que nos outros grupos foi observada uma elevação superior, no D60 os grupos controle e SIM pode-se notar uma elevação nos escores de reatividade, enquanto o grupo PRE manteve-se praticamente estável e o grupo PRO abaixou as médias, mantendo a reatividade baixa no D90, indicando adaptação ao manejo de confinamento, os grupos controle e PRE também diminuíram as médias de reatividade no D90, já o grupo SIM manteve altos índices de reatividade (Figura 1). Segundo Sant'Anna et al. (2015) a seleção genética do gado zebuíno no Brasil não é eficiente no critério de temperamento dos animais. 


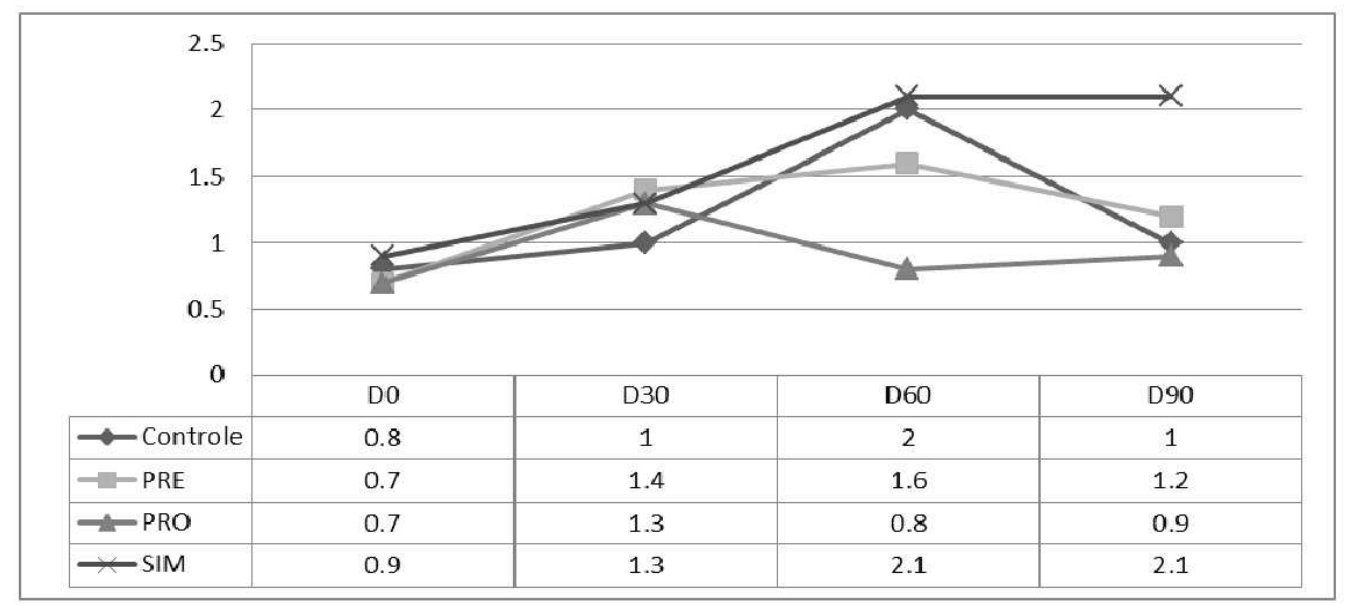

FIGURA 1: Média do cálculo de reatividade de novilhas nelores confinadas recebendo na dieta prebiótico (PRE), probiótico (PRO), simbióse (SIM) e grupo controle.

Segundo Rueda et al. (2015) o estresse também influencia negativamente na reprodução, animais mais reativos tendem a ter uma taxa de prenhes menor quando comparados com animais menos reativos. Nota-se uma relação da figura $1 \mathrm{com}$ a tabela 3 nos animais do grupo SIM, observa-se na figura 1 que as novilhas apresentam alta reatividade, indicando não adaptação ao confinamento, na tabela 3, no D90 foi encontrada diferença estatística no grupo SIM em relação aos demais grupos, os níveis de cloretos na corrente sanguínea estão superiores aos demais, que segundo França e Rodriguez (1997) é um indicativo de estresse acumulado, apontando assim, uma relação do método de cálculo de reatividade e os parâmetros bioquímicos.

Ceballos et al. (2016) afirmam que o manejo rotação de pastagens em bovinos proporciona maior tranquilidade para os animais em longo prazo, os autores creditam isso pela interação do ser humano com os animais, desta forma observa-se no presente estudo que os animais dos grupos controle, PRE e PRO apresentaram baixa reatividade no D90.

\section{CONCLUSÃO}

Nas condições experimentais utilizadas, o uso de prebióticos, probióticos e simbióticos não favoreceram a homeostase e a adaptação ao confinamento de novilhas da raça Nelore, quando observamos os parâmetros bioquímicos de glicose, cortisol, fósforo e cloretos e os parâmetros hematológicos de leucócitos, neutrófilos, linfócitos, hemácias, hemoglobinas, hematócritos, proteínas totais, albuminas e globulinas. A planilha de reatividade mostrou-se uma ferramenta útil na observação de reatividade, acompanhando os picos de cloretos no plasma sanguíneo nos animais mais estressados.

\section{REFERÊNCIAS}

AGUILAR, N. M. A. Avaliação da reatividade de bovinos de corte e sua relação com caracteres reprodutivos e produtivos, 2007. 80 f. Dissertação (Mestrado em Zootecnia) - Universidade Estadual Paulista. 
APPELT, M. D.; NUNES, R. V.; POZZA, P. C.; SILVA, W. T. M.; VENTURI, I.; NUNES, C. G. V. Níveis de probiótico em ração de origem animal e vegetal para frangos de corte. Revista Brasileira de Zootecnia, v. 39, n. 4, p. 765 - 771. 2010.

BITTAR, C. M. M.; SILVA, F. L. M.; PAULA, M. R.; SILVA, J. T.; GALLO, M. P.; OLTRAMARI, C. E., et al. Desempenho e parâmetros sanguíneos de bezerros em sistemas de desaleitamento precoce suplementados com probióticos de bactérias ruminais. Revista Brasileira de Saúde Animal, v. 17, n. 2, p. $249-261$, abr - jun. 2016. http://www.scielo.br/scielo.php?pid=S151999402016000200249\&script=sci_abstract\&tlng=pt> doi: $10.1590 / \mathrm{S} 1519$ 99402016000200012.

BUDIÑO, F. E. L.; THOMAZ, M. C.; KRONKA, R. N.; PIZAURO JUNIOR, J. M.; SANTANA, A. E.; TUCCI, F. M. et al., Influencia da adição de probiótico e/ou prebiótico em dietas de leitões desmamados sobre as atividades das enzimas digestivas e parâmetros sanguíneos. Acta Scientiarum Animal Sciences, v. 26, n. $4, \quad \begin{array}{llllll} & \text { p. } & 529 & - & 536 . & 2004 .\end{array}$ http://periodicos.uem.br/ojs/index.php/ActaSciAnimSci/article/view/1743> doi: 10.4025/actascianimsci.v26i4.1743.

CEBALLOS, M. C.; GÓIS, K. C. R.; SANT'ANNA, A. C.; PARANHOS da COSTA, M. $\mathrm{J}$. R. Frequent handling of grazing beef cattle maintained under the rotational stocking method improves temperament over time. Animal Production Science, v.56, p.10.1071, 2016. <http://www.publish.csiro.au/AN/AN16025> doi: 10.1071/AN16025.

CHAUD, S. G.; SGARBIERI, V. C.; VICENTE, E.; SILVA, N.; ALVEZ, A. B.; de MATOS, J. A. R. Influencia de Frações da Parede Celular de Leveduda (Saccharomyces cereviseae) sobre os índices séricos de glicose e lipídios, microbiota intestinal e produção de ácidos graxos voláteis (AGV) de cadeias curtas de ratos em crescimento. Ciếncia e Tecnologia de Alimentos, v. 27, n. 2, p. $338-$ 348, Abr- Jun. 2007. <http://www.scielo.br/scielo.php?script=sci_arttext\&pid=S010120612007000200023 > doi: 10.1590/S0101-20612007000200023.

CHIQUIERI, J.; SOARES, R. T. R. N.; HURTADONERY, V. L.; CARVALHO, E. C. Q.; COSTA, A. P. D. Bioquímica sanguínea e altura das vilosidades intestinais de suínos alimentados com adição de probióticos, prebióticos e antibióticos. Revista Brasileira de Saúde e Produção Animal, v. 8, n. 2, p. $97-<104,2007$. < http://revistas.bvs-vet.org.br/rbspa/article/viewFile/9467/10185>.

FMVZ - Faculdade de Medicina Veterinária e Zootecnia. Universidade Estadual Paulista- UNESP, Botucatu- SP. Hemograma. Disponível em: http://www.fmvz.unesp.br/Home/Departamentos/DCV/profareginakiomitakahira/valref .pdf. Acesso em: 30/11/2016.

FRANÇA, A. C. L.; RODRIGUES, A. L. Stress e trabalho: Guia Básico com Abordagem Psicossomática. 1997. 181p.

GONZÁLEZ, F. H. D.; CONCEIÇÃO, T. R.; SIQUEIRA, A. J. S; LA ROSA, V. L. Variações sanguínea da ureia, creatina, albumina e fósforo em bovinos de corte no 
Rio Grande do Sul. A Hora Veterinária, v. 20, p. 59 - 62. 2000. https://www.ufrgs.br/lacvet/restrito/pdf/gonzalez_perfil_butia.pdf.

JUNQUEIRA, O. M.; CAMARGO FILHO, B.; ARAÚJO, L. F.; ARAÚJO, C. S. S.; SAKOMURA, N. K. Efeitos das fontes e níveis de sódio, cloro e potássio e da relação $(\mathrm{Na}+\mathrm{K}) / \mathrm{Cl}$, sobre o desempenho e características do plasma sanguíneo de poedeiras comerciais. Revista Brasileira de Zootecnia, v. 29, n. 4, p. $1110-1116$. 2000. < http://www.scielo.br/pdf/rbz/v29n4/5626.pdf>. doi: 10.1590/S151635982000000400023.

MAGNANI, M.; CASTRO-GÓMEZ, R. J. H. Beta-Glucana de Saccharomyces cerevisiae. Semina: Ciências Agrárias, v. 29, n. 3, p. 631 - 650, 2008. < http://www.uel.br/proppg/portal/pages/arquivos/pesquisa/semina/pdf/semina_29_3_1 9_15.pdf >. doi: 10.5433/1679-0359.

MARDEN, J. P.; JULEIN, C.; MONTEILS, V.; AUCLAIR, E.; MONCOULON, R.; BAYOURTHE, C. How does live yeast differ from sodium bicarbonate to stabilize ruminal $\mathrm{pH}$ in high-yielding dairy cows? Journal of Dairy Science, v. 91, n. 9, p.3528-3535, 2008. <http://www.journalofdairyscience.org/article/S00220302(08)71067-1/pdf>. doi: 10.3168/jds.2007-0889.

RUEDA, P.M.; SANT'ANNA, A. C.; VALENTE, T. S.; PARANHOS da COSTA, M. J. R. Impact of the temperament of Nellore cows on the quality of handling and pregnancy rates in fixed-time artificial insemination. Livestock Science, v. 177, p. $189 \quad-\quad 195, \quad 2015 .<<$ http://www.sciencedirect.com/science/article/pii/S1871141315002218>. doi: 10.1016/j.livsci.2015.04.021.

SANT'ANNA, A. C.; PARANHOS da COSTA, M. J. R.; BALDI, F. RUEDA, P. M.; ALBULQUERQUE, L. G. Genetic Associations between flight speed and growth traits in Nellore cattle. Journal of Animal Science, p. 3427 - 3432. 2015. <file://C:/Users/ACER/Downloads/jas-90-10-3427.pdf>>. doi: 10.2527/jas.20115044.

TOGASHI, C. K.; FONSECA, J. B.; SOARES, R. T. R. N.; SOUZA, C. L. M. Determinação de Níveis de Metionina+Cistina para Poedeira Semi-Pesadas Alimentadas com Ração Contendo Levedura Seca (Saccharomyces serevisiae). Revista Brasileira de Zootecnia, v. 31, n. 3, p. 1426 - 1433. 2002 . < http://www.scielo.br/scielo.php?pid=S1516-

35982002000600014\&script=sci_abstract\&tlng=pt>. doi: $\quad$ 10.1590/S151635982002000600014.

VÁSQUEZ, E. F. A.; HERRERA, A. P. N. Concentração plasmática de cortisol, ureia, cálcio e fósforo em vacas de corte mantidas a pasto suplementadas com levedura de cromo durante a estação de monta. Ciência Rural, v. 33, n. 4, 743 - 747, 2003. < http://www.scielo.br/scielo.php?script=sci_arttext\&pid=S0103-84782003000400025>. doi: 10.1590/S0103-84782003000400025. 